\title{
Synchrotron strain scanning for residual stress measurement in cold-drawn steel rods
}

J Ruiz-Hervias*, J M Atienza, and M Elices

Departamento de Ciencia de Materiales, Universidad Politécnica de Madrid, Madrid, Spain

\begin{abstract}
Cold-drawn steel rods and wires retain significant residual stresses as a consequence of the manufacturing process. These residual stresses are known to be detrimental for the mechanical properties of the wires and their durability in aggressive environments. Steel makers are aware of the problem and have developed post-drawing processes to try and reduce the residual stresses on the wires. The present authors have studied this problem for a number of years and have performed a detailed characterization of the residual stress state inside cold-drawn rods, including both experimental and numerical techniques. High-energy synchrotron sources have been particularly useful for this research. The results have shown how residual stresses evolve as a consequence of cold-drawing and how they change with subsequent post-drawing treatments. The authors have been able to measure for the first time a complete residual strain profile along the diameter in both phases (ferrite and cementite) of a cold-drawn steel rod.
\end{abstract}

Keywords: cold-drawing, steel wires, residual stress, synchrotron

\section{INTRODUCTION}

Steel wires and rods are widely used in industry, as cables for bridges and as reinforcements in prestressed concrete structures and car tyres (steel cords). Prestressing steel wires have a lamellar pearlitic microstructure $(0.8 \% \mathrm{C})$ with two phases: ferrite (approx. 90\% vol.) and cementite (approx. 10\% vol.). They are fabricated by cold-drawing, which consists of reducing the diameter in a step-wise manner. To this end the initial rod is pulled through a series of conical dies with decreasing diameters, until the final wire is obtained. As a consequence, a sharp wire texture is developed, the ferrite/cementite lamellae are elongated along the wire axis, and the interlamellar spacing is decreased. In addition, residual stresses are generated owing to the heterogeneous deformation imparted to the rod during the process.

*Corresponding author: Universidad Politécnica de Madrid, Departamento de Ciencia de Materiales, ETSI Caminos, c/ Profesor Aranguren, s/n E-28040 Madrid, Spain. email:jr@mater.upm.es
It is known that the mechanical behaviour of the wires (tensile strength and toughness) is mainly controlled by the microstructure $[\mathbf{1 - 3}]$. On the other hand, the durability (fatigue and environmentally assisted cracking) is also influenced by the residual stresses generated during drawing. This was repor-ted a long time ago, but published results for these particular steels were barely quantitative $[\mathbf{4}, \mathbf{5}]$.

The durability of prestressing steel wires in aggressive environments (i.e. bridges near or on the coastline) is dominated by two factors: surface defects and residual stresses at the surface region [6]. In commercial products, little can be done about surface defects because they come from the initial rod. Some of them are eliminated during cold-drawing, but most remain (stretched along the wire axis). Consequently, it is more productive to focus on residual stress control. The steelmakers are aware of the problem and they have developed post-drawing treatments to improve the durability of prestressing wires in aggressive environments. Their approach was purely empirical though, because reliable residual stress data were lacking. The question is how to 'design' the best residual stress 
profile in order to enhance wire durability in aggressive environments.

The development of powerful experimental techniques (i.e. neutron and synchrotron diffraction) has allowed the measurement of residual strains in a non-destructive fashion inside prestressing steel wires and rods [6-8]. The present authors have studied this problem for a number of years and have carried out a detailed characterization of the residual stress state inside cold-drawn rods, including both experimental and numerical techniques $[6,9$ 12]. The results have shown how residual stresses evolve as a consequence of cold-drawing and how they change with subsequent post-drawing treatments. The use of high-energy synchrotron sources has been particularly useful for this research. Residual strain scanning has allowed the obtaining of reliable quantitative residual stress profiles with high spatial resolution. In particular, the authors have been able to measure, for the first time, a complete residual strain profile along the diameter in both phases (ferrite and cementite) of a cold-drawn steel rod $[\mathbf{9}]$. In addition, the experimental data were used to validate the results of numerical simulations of the cold-drawing process $[\mathbf{9}, \mathbf{1 0}]$. Moreover, these results have been successfully correlated with the mechanical performance and durability of these steels, particularly with fracture owing to hydrogen embrittlement [13].

The aim of the current paper is to show the usefulness of synchrotron radiation to improve existing cold-drawing processes and post-drawing treatments by using high-resolution strain scanning. Results obtained by the authors using synchrotron radiation will be updated and summarized. The problems associated with residual stress characterization in prestressing steel wires and rods will be addressed. In addition, data obtained from finite element modelling of the cold-drawing process will be compared with experimental results.

\section{MATERIALS}

The results corresponding to two different steel rods are presented in this paper. Both have a fully pearlitic microstructure $(0.8 \% \mathrm{C})$ but they have different diameters and they have been subjected to different cold-drawing processes. The first one was colddrawn in one pass from a $20 \mathrm{~mm}$ diameter bar up to a final diameter of $18 \mathrm{~mm}$ (a 20 per cent reduction in section). True strain given by drawing reaches 0.2 , as calculated by $2 \ln \left(\Phi_{0} / \Phi_{\mathrm{f}}\right)$, where $\Phi_{0}$ and $\Phi_{\mathrm{f}}$ are the initial $(20 \mathrm{~mm})$ and final $(18 \mathrm{~mm})$ diameters, respectively. The drawing process was thoroughly controlled and the bars were kept straight during the whole process. Further details are given in reference $[\mathbf{1 4}]$. The second material is a commercial prestressing steel rod. In this case, the initial rod (12 mm diameter) is subjected to a multi-step drawing process (eight steps in total) until the diameter corresponding to the final product $(5.2 \mathrm{~mm})$ is reached. Total section reduction is 81 per cent and true strain is 1.7. Further details are given in reference [11]. From this material, three different samples were studied. They have the same microstructure and very similar mechanical properties and they differ in their residual stress state, as outlined below.

1. 'As-drawn': resulting from cold-drawing without any post-drawing treatment.

2. 'Stabilized': subjected to a thermo-mechanical treatment after drawing with the aim of reducing the residual stress level.

3. 'Rolled': stabilized plus surface rolling (the procedure is described in reference [6]). This produces a small plastic deformation on the wire surface, analogous to a 'massage'. Compressive residual stresses can be induced on the wire surface by this procedure.

The microstructure in both materials is fully pearlitic, as corresponds to the eutectoid point $(0.8 \% \mathrm{C})$. In the micrographs obtained by scanning electron microscopy (SEM) (Fig. 1), both the initial (12 $\mathrm{mm}$ diameter) and the 'as-drawn' rod (5.2 $\mathrm{mm}$ diameter) corresponding to the commercial prestressing steel rod are shown. It can be seen that the microstructure is fairly isotropic in the initial rod, with the ferrite/cementite lamellae randomly oriented, as shown in Figs 1 (a) and (b). After drawing, the lamellae are considerably thinner (interlamellar spacing around $100 \mathrm{~nm}$ ) and mainly aligned in the drawing direction, which coincides with the rod axis, as seen in Figs 1(c) and $(\mathrm{d})$.

For the sake of comparison, the $18 \mathrm{~mm}$ rod sample will be referred to as the lightly drawn material and the $5.2 \mathrm{~mm}$ prestressing rods will be called severely drawn materials.

\section{EXPERIMENTAL PROCEDURE AND RESULTS}

High-energy X-ray diffraction (XRD) experiments were performed at the ID15A beamline of the ESRF (Grenoble, France). The beamline was operated with a superconducting wavelength shifter (SCWS) as insertion device and a critical energy of $95.8 \mathrm{keV}$. 

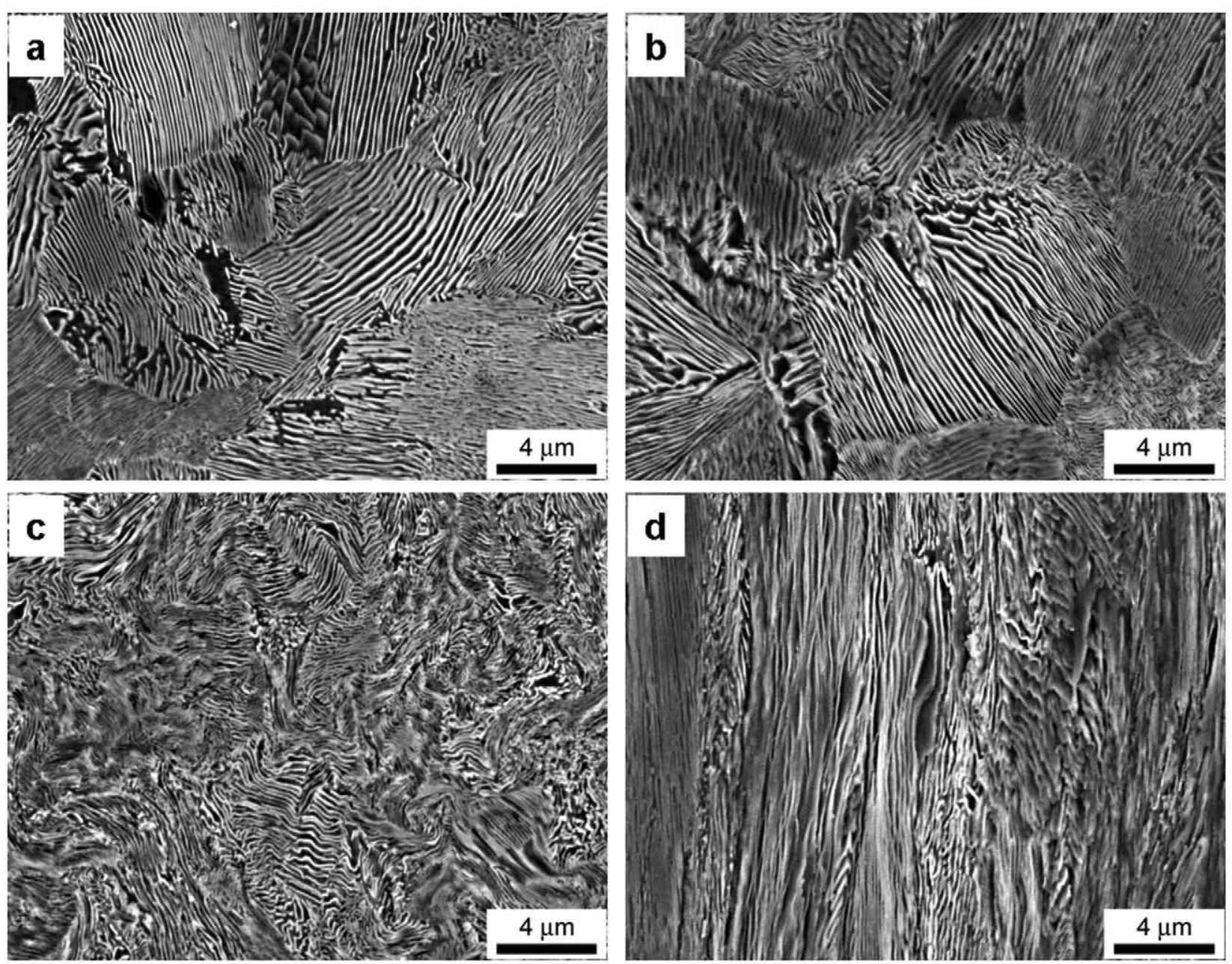

Fig. 1 Microstructure of the severely drawn pearlitic steel rod. Initial rod before drawing: (a) transverse cross-section, (b) longitudinal section; and 'as-drawn' rod: (c) transverse crosssection, and (d) longitudinal section. The drawing direction (parallel to the rod axis) is perpendicular to the paper in (a) and (c) and lies in the paper (vertical direction) in (b) and (d). The light features correspond to the cementite and the dark regions between cementite lamellae are occupied by ferrite, which is etched by the reagent employed (Nital 2 per cent)

The diffraction patterns were collected in white beam configuration (energy dispersive). In the experiments with the lightly drawn material, one Ge detector was used (resolution $\Delta \tilde{\mathrm{E}} / \mathrm{E} \simeq 0.001$ ) whereas two detectors (horizontal and vertical) were used with the severely drawn material. This means that two directions of strain could be measured at the same time in the latter. As regards the scattering angle, it was set to $3.5^{\circ}$ and $5^{\circ}$ for the lightly and severely drawn materials, respectively.

In all samples strain scanning was performed in the three principal directions of the rods (axial, radial, and hoop) along a complete rod diameter. The scanning step was set to $0.75 \mathrm{~mm}$ in the lightly drawn material. In this case, the incident slit dimensions were varied between $0.15 \times 0.15$ and $0.4 \times 0.4 \mathrm{~mm}^{2}$ whereas the receiving slit was set to $0.05 \mathrm{~mm}$ width. In the severely drawn samples, the incident slit dimensions were $0.1 \times 0.1 \mathrm{~mm}^{2}$, the receiving slit was set to $0.1 \mathrm{~mm}$, and the scanning step was $0.1 \mathrm{~mm}$. Consequently, the gauge volume has the form of an elongated diamond, as shown in Fig. 2.

Strain analysis can be carried out by substituting the photon energy in Bragg's law

$$
\frac{1}{E_{\mathrm{hkl}}}=\frac{2}{h c} d_{\mathrm{hkl}} \sin \theta
$$

where $h$ is the Planck's constant, $c$ is the speed of light in vacuum, $2 \theta$ is the scattering angle, and $d_{\mathrm{hkl}}$ and $E_{\mathrm{hkl}}$ are respectively the lattice spacing and energy of the hkl reflection. The strain for every (hkl) 


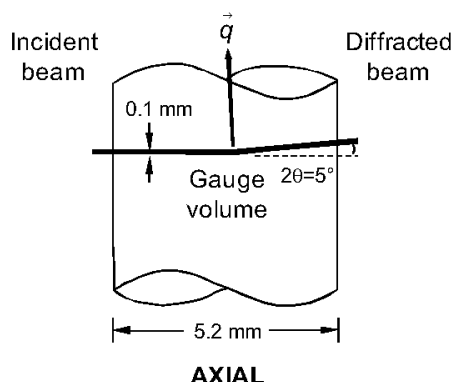

AXIAL

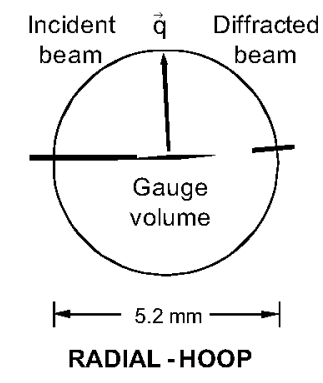

Fig. 2 Schematic representation of the gauge volume employed in the synchrotron strain scanning experiments (severely drawn materials) for the three directions: axial, radial, and hoop (all dimensions are scaled)

set of planes can be obtained from the variation in $d$-spacing

$$
\varepsilon_{\mathrm{hkl}}=\frac{d_{\mathrm{hkl}}-d_{\mathrm{hkl}}^{0}}{d_{\mathrm{hkl}}^{0}}=\frac{E_{\mathrm{hkl}}^{0}-E_{\mathrm{hkl}}}{E_{\mathrm{hkl}}}
$$

where $\varepsilon_{\mathrm{hkl}}$ is the longitudinal strain in the direction of the scattering vector, and $d_{\mathrm{hkl}}^{0}$ and $E_{\mathrm{hkl}}^{0}$ are the unstressed lattice spacing and corresponding energy of the hkl reflection, respectively. Once the strain components are calculated by equation (2), the stresses are computed through the following equation

$$
\sigma_{i j}=\frac{1}{S_{2} / 2}\left[\varepsilon_{i j}-\frac{S_{1}}{S_{2} / 2+3 S_{1}} \delta_{i j} \varepsilon_{k k}\right]
$$

where $\delta_{i j}$ is the Kronecker's delta function, $S_{1}$ and $S_{2} / 2$ are the diffraction elastic constants (DEC) and the summation convention is employed (repeated index). The DECs employed for the ferrite $\{110\}$ reflection were calculated from the single crystal elastic constants (Kröner model): $S_{1}=-1.175 \times$ $10^{-12} \mathrm{~m}^{2} / \mathrm{N}, S_{2} / 2=5.925 \times 10^{-12} \mathrm{~m}^{2} / \mathrm{N}$. In the case of the cementite phase, data reported in the literature were used for the $\{122\}$ reflection [9]: $S_{1}=-1.469 \times 10^{-12} \mathrm{~m}^{2} / \mathrm{N}, S_{2} / 2=7.119 \times 10^{-12} \mathrm{~m}^{2} / \mathrm{N}$.

A moderate (110) fibre axis texture was found in the lightly drawn material [9]. In this case, single peak analysis was performed in both phases. A pseudo-Voigt function was used for analysis of the $\{110\}$ reflection of the ferrite phase. On the other hand, strain scanning in the cementite phase was performed using the $\{122\}$ reflection. This reflection was chosen because it does not present any overlapping, which makes the analysis easier. An example of the collected diffractograms (axial component at the rod core) is shown in Fig. 3 [9]. The ferrite peaks are much more intense than the cementite ones,

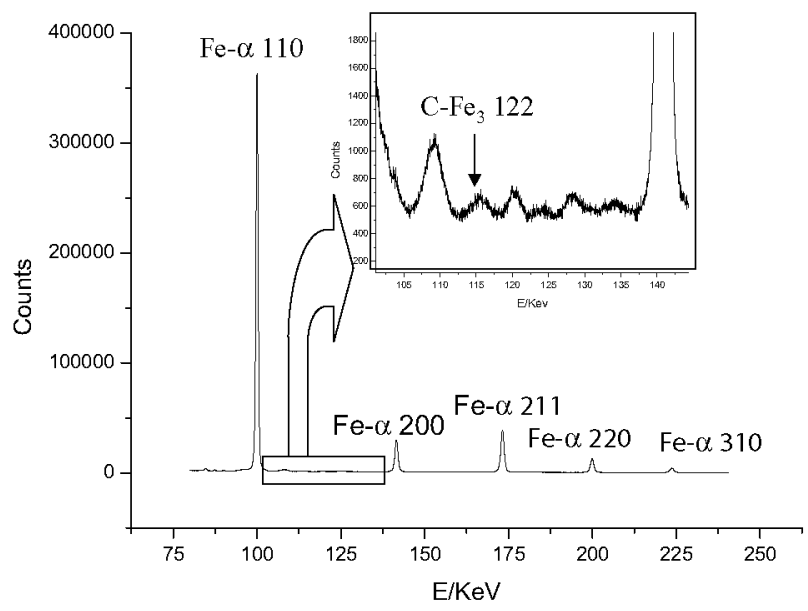

Fig. 3 Example of the collected diffractograms at the ID15A beamline (ESRF) in energy scale (axial component at the rod core). The ferrite reflections are pointed out and the inset shows the cementite reflections in the spectral range 100 to $150 \mathrm{keV}$. The arrow corresponds to the 122 reflection, which does not overlap with any other reflection, and was chosen for the strain analysis (Reprinted from [9] with permission from Elsevier.)

which are almost hidden in the background. The cementite reflections in the spectral range between 100 and $150 \mathrm{keV}$ (between the ferrite 110 and 200 reflections) are shown in the insert. The $\{122\}$ cementite reflection is located at $115 \mathrm{keV}$ in this case (which corresponds to a lattice spacing of $1.766 \AA$ ). The stress-free lattice parameter for ferrite was measured from iron filings and for cementite it was calculated by assuming self-equilibrium (details are given in reference [9]). Residual macrostresses in the pearlitic material were determined by using the ferrite and cementite phase stresses, weighted with their relative percentages (rule of mixtures, in this case 90 percent ferrite and 10 percent cementite). Ferrite and cementite stresses are depicted in Fig. 4. The macrostress profile in the axial direction is shown in Fig. 5, together with the ferrite and cementite phase stresses. To the authors' knowledge, this is the first time that a complete residual stress profile is reported in both phases (ferrite and cementite) of a cold-drawn eutectoid rod.

The severely drawn material shows a sharp (110) fibre axis texture [11]. The effect of texture can be seen in Fig. 6, where typical diffraction patterns for the radial-hoop and axial directions of the rods are depicted [15]. In the axial direction, most of the ferrite reflections except (110) have almost disappeared. Full pattern structure refinement (LeBail) was performed and the calculated intensities, the difference between observed and calculated values and the ferrite and cementite reflections, are also 


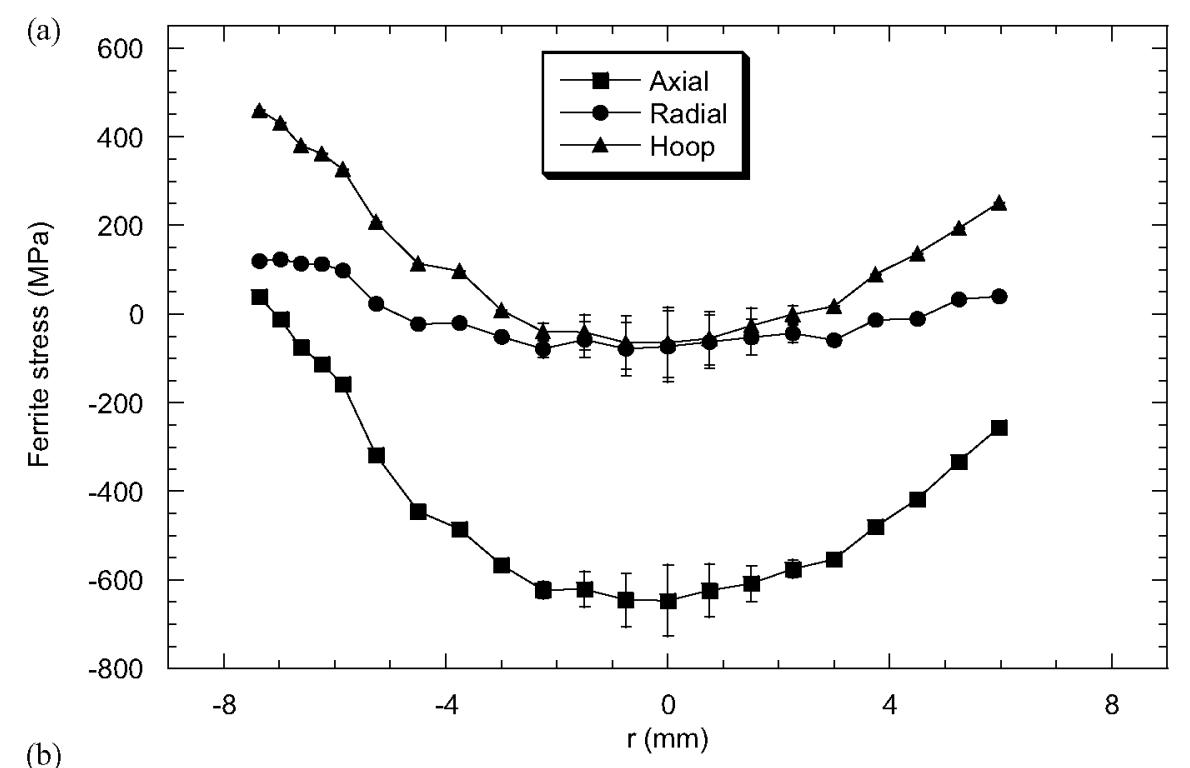

(b)

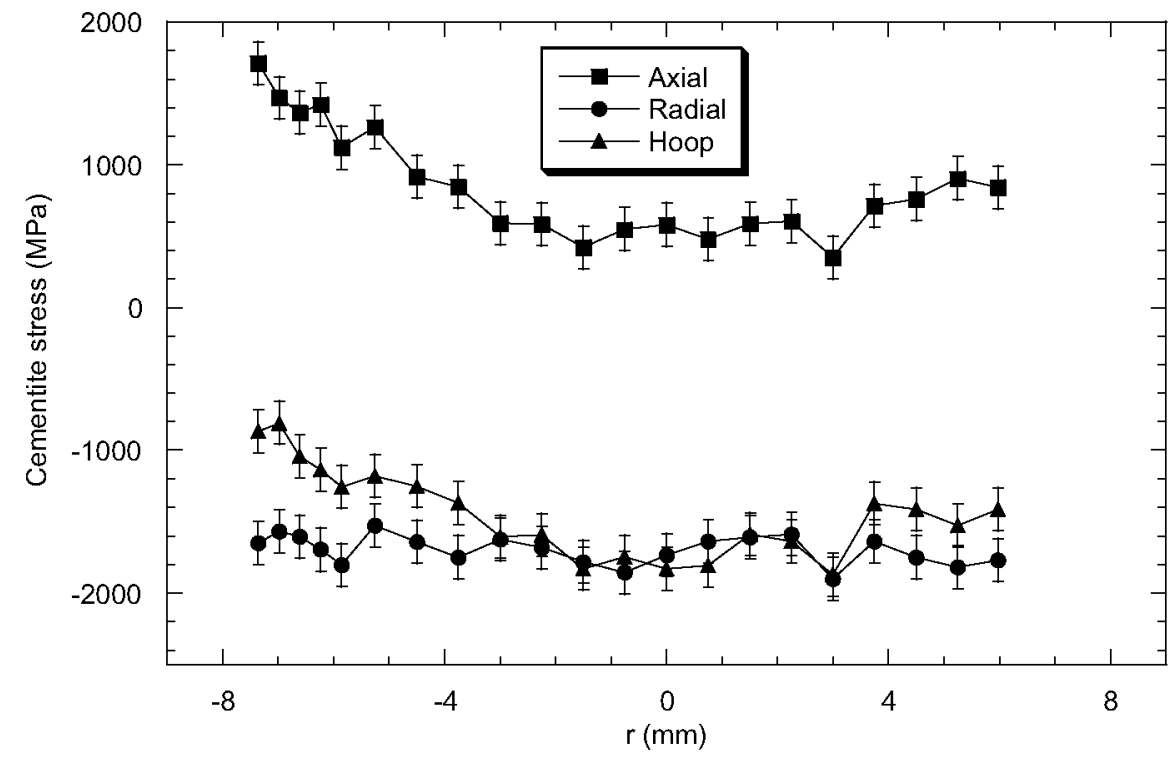

Fig. 4 Stress profiles obtained from synchrotron X-ray diffraction in the light drawn material: (a) ferrite and (b) cementite phase (Reprinted from [9] with permission from Elsevier.)

shown in Fig. 6. The signal from the cementite phase is very low, and the (122) reflection has disappeared, as shown in the inset in Fig. 6(b). Consequently, in this material only results for the ferrite phase are reported. Full pattern refinement was performed in the radial and hoop directions, whereas single peak fitting using the 110 reflection was carried out in the axial direction.

In this case, the ferrite stress-free lattice parameter was not measured. As the residual stress state in the central portion of the 'stabilized' sample is hydrostatic, an approximate $d_{0}$ was calculated by averaging the measured lattice spacing in the three principal directions corresponding to this region [11]. The calculated residual stress profiles for the three samples studied are given in Figs 7 to 9 [15]. The use of synchrotron radiation allows the obtaining of data with high spatial resolution $(0.1 \mathrm{~mm}$ steps), which in turn produces very smooth stress profiles.

For the 'as-drawn' sample, the residual stress profiles are similar to those measured in the ferrite phase of the lightly drawn material (compare Figs 4(a) and 7). Axial residual stresses are compressive at the rod centre, with values close to $-800 \mathrm{MPa}$, and they increase their value towards the surface. The hoop stresses, on the other hand, are mostly tensile, with values ranging from 0 to $300 \mathrm{MPa}$. As regards the radial stresses, they are negligible and almost constant along the rod diameter.

The stabilizing treatment is very effective in reducing the residual stresses generated by colddrawing (compare Figs 7 and 8). The residual stress 


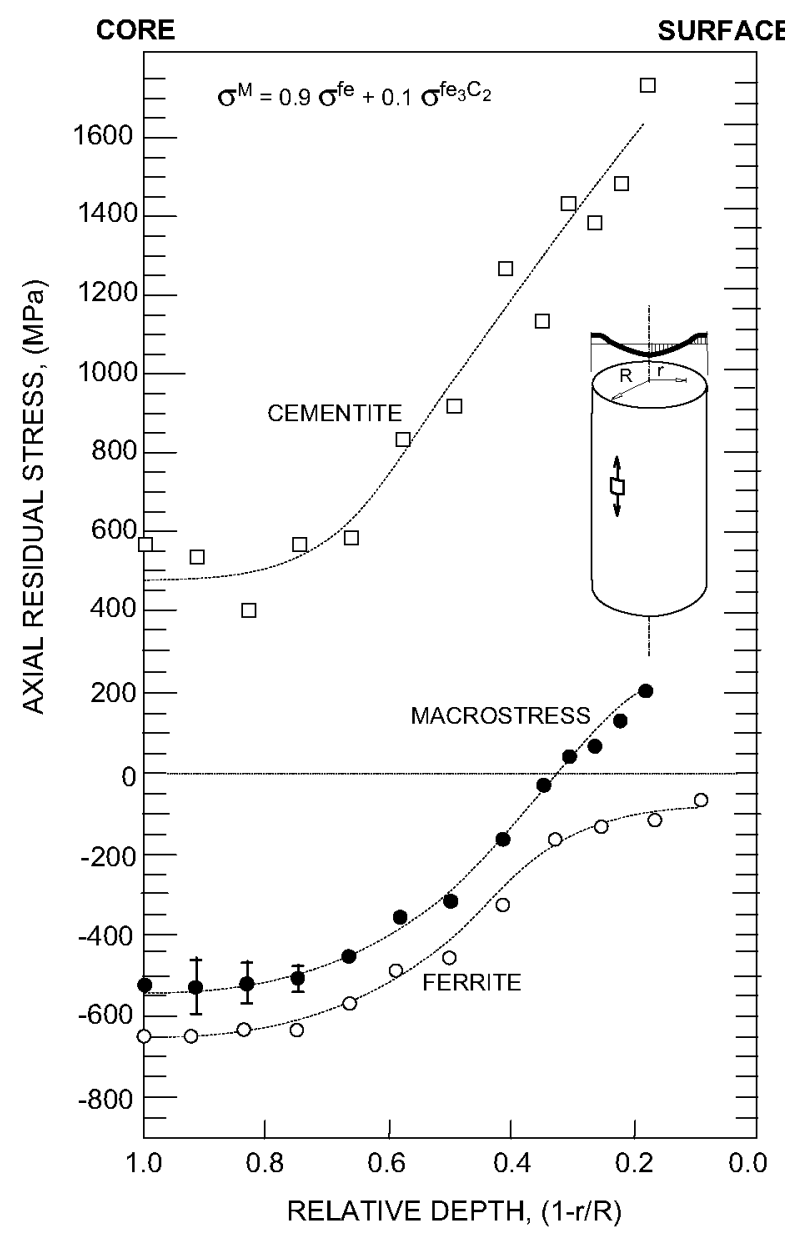

Fig. 5 Axial residual stresses in the ferrite and cementite phases of the lightly drawn steel rod as measured by synchrotron radiation. Macro-stresses were computed from the phase stresses weighted with their relative volume fractions ( 90 per cent ferrite, 10 per cent cementite) [9]. In this case the lines only serve as a guide for the eye (Reprinted from [9] with permission from Elsevier.)

field in the 'stabilized' sample is almost constant along the rod diameter. This is particularly noticeable for the axial component, where the high compressive stresses at the rod centre almost disappear, and the resulting profile is much smoother than in the 'as-drawn' sample.

On the other hand, the 'rolled' samples show a different residual stress field (see Fig. 9). The axial stresses are tensile at the rod centre (between 200 and $250 \mathrm{MPa}$ ) and tend to compressive values at the surface. The hoop and radial stresses have a similar behaviour, with tensile values at the rod centre (maximum around $100 \mathrm{MPa}$ ).

The residual stress profiles corresponding to the ferrite phase are clearly unbalanced in the 'asdrawn' and 'rolled' samples (see Figs 7 and 9). This is attributed to the fact that the contribution of the cementite phase is not accounted for. The situation is improved in the 'stabilized' sample, owing to the small residual stress values obtained. For example, in the 'as-drawn' sample the axial residual stresses are always negative, whereas the hoop stresses are always positive. Cementite phase stress profiles obtained in the lightly drawn material show that the axial stresses oscillate between 1000 and $2000 \mathrm{MPa}$, whereas the radial and hoop stresses range from -2000 to $-1000 \mathrm{MPa}$ [9]. If a similar behaviour is assumed for the current case, the cementite stresses would help to achieve the balance, because the axial profile would be displaced up and the hoop one would be displaced down.

The stress distribution in the rod after drawing was computed by finite element modelling. A numerical model using the code ABAQUS was developed for this purpose [16]. An elastoplastic law with strain hardening was chosen to model the rod behaviour. The experimental stress-strain curve of the initial rod (before drawing) was used as the constitutive equation. The drawing process was simulated by forcing the wire to pass through the die and imposing the displacement of the front end of the wire. The die was modelled as an elastic material. The contact between the wire and the die was reproduced with a Coulomb friction coefficient (several calculations were performed with values ranging from 0.01 to 0.2 ). Residual stresses were calculated at the end of the process, when the whole rod had passed through the die, in a zone where the stationary state had been reached.

A correction was employed to include the anisotropy of drawn pearlite. As a first approximation to represent the anisotropy, the Hill yield criterion was chosen. A user's subroutine in ABAQUS was defined to change the yield stresses in the different planar directions according to the progressive alignment of the cementite lamellae $[\mathbf{1 0}, \mathbf{1 6}]$.

The axial macrostresses in the lightly drawn material (calculated from the synchrotron measurements) are compared with the finite element simulations in Fig. $10[\mathbf{1 0}]$. The agreement between experimental and numerical results is good, especially when the anisotropy is taken into account. The numerical model without anisotropy overestimates the real values of residual macrostresses particularly at the rod centre and at the surface.

Figure 11 shows the calculated profiles of axial macro-residual stresses along the rod diameter as a function of the relative depth in the as-drawn, stabilized, and rolled rods. If the calculated stress profiles are compared with the experimental results for the ferrite phase (especially for the as-drawn and rolled rods) it can be seen that the shape is similar 
(a)

Radial \& hoop directions

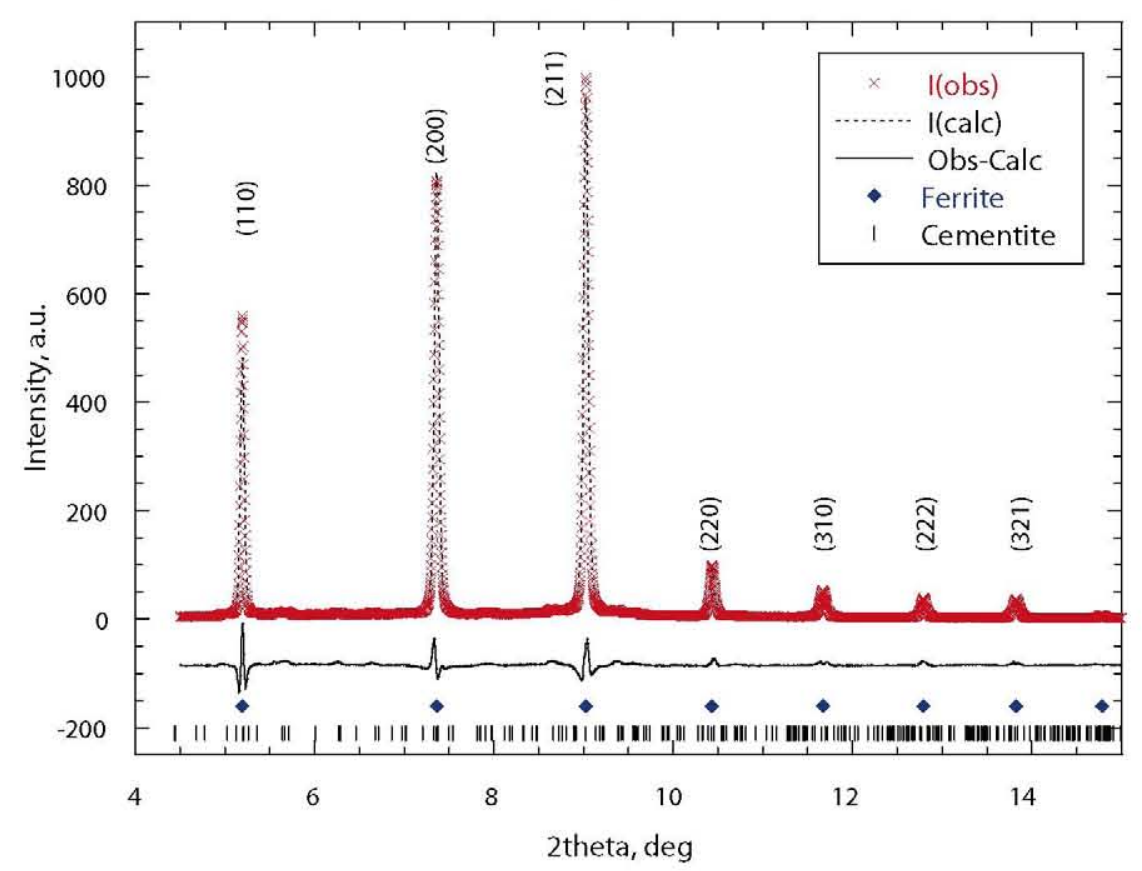

(b)

Axial direction

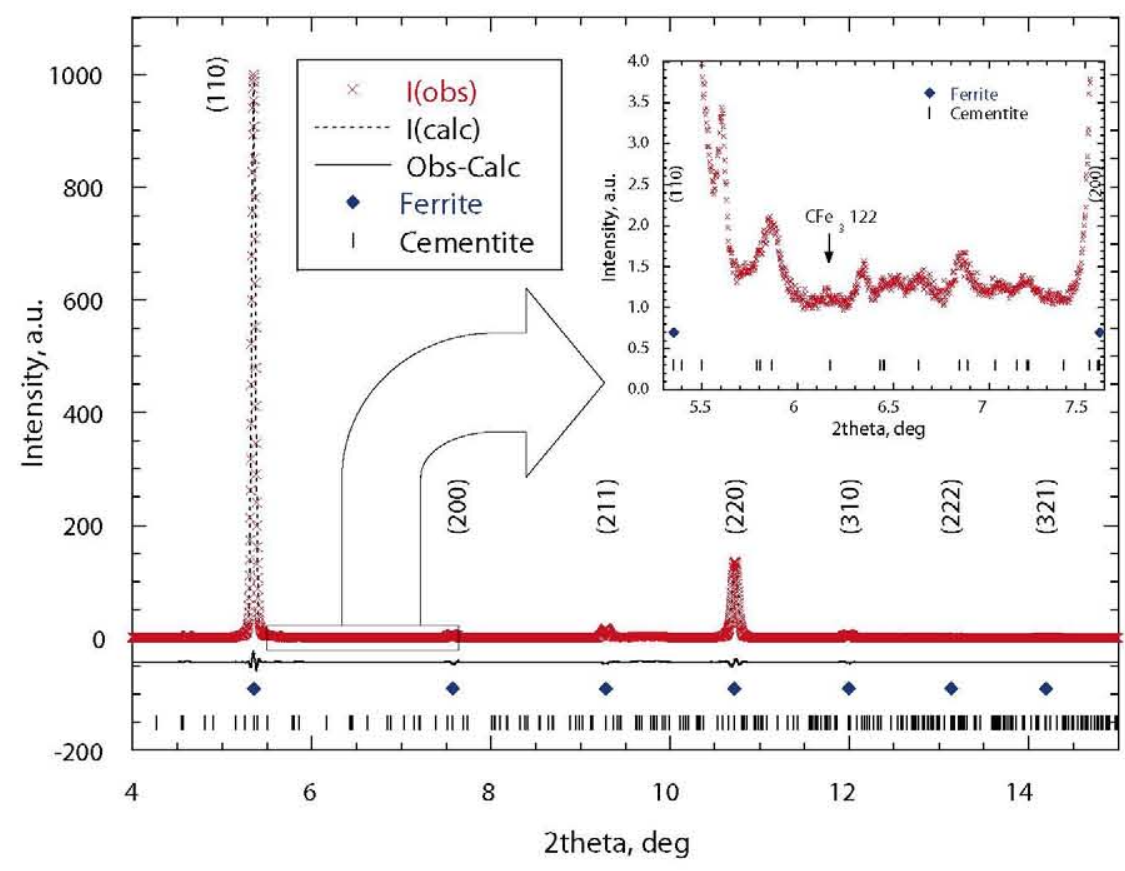

Fig. 6 Example of the collected diffractograms at the ID15A beamline (ESRF) for the severely drawn material [15]. The original results were converted to pseudo constant wavelength data (using a $0.180 \AA$ wavelength): (a) hoop and radial components, and (b) axial component. The observed and calculated intensities are shown, along with the difference curve and the ferrite and cementite reflections. The inset in (b) shows that the cementite 122 reflection has disappeared (axial component)

(compare Fig. 11 with Figs 7 and 9). The results are different though, because cementite strains could not be measured in this case.
The hydrogen susceptibility of the three sets of rods was measured by using the International Federation for Prestressing, ammonium thiocyanate (FIP) test. Six 


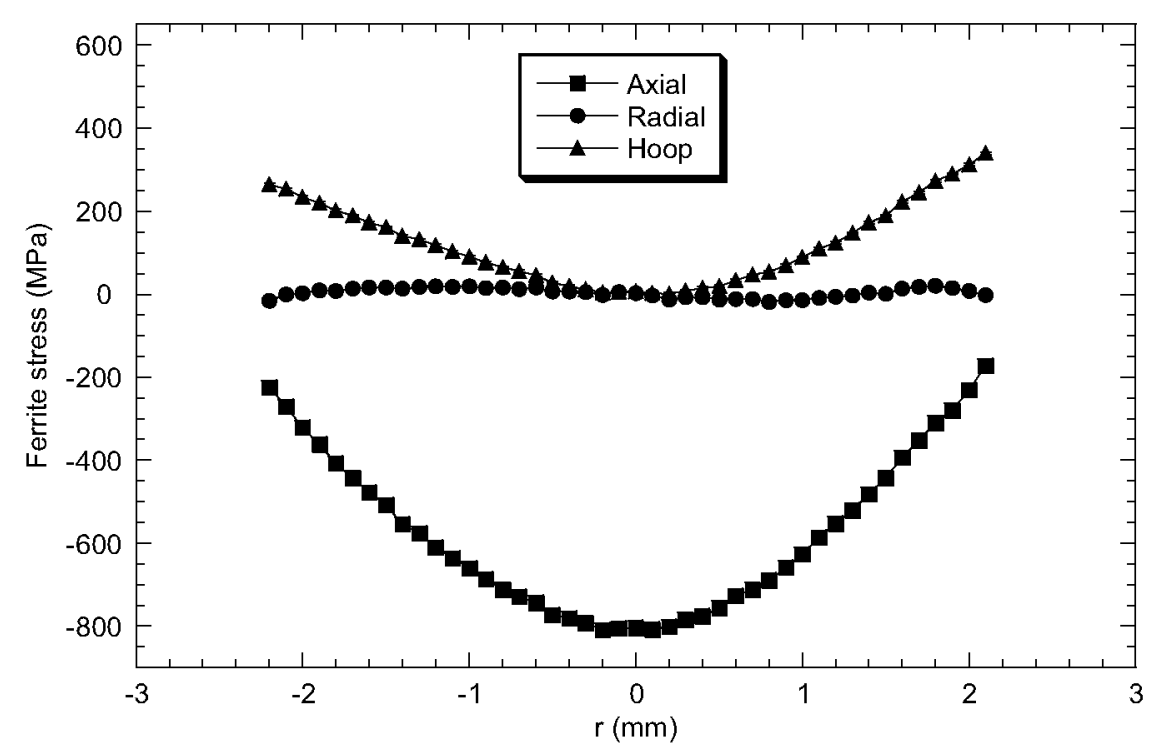

Fig. 7 Residual stress profiles obtained from the synchrotron measurements in the ferrite phase of the 'as-drawn' steel rod (severely drawn material) [15]. Error bars are smaller than the symbols employed

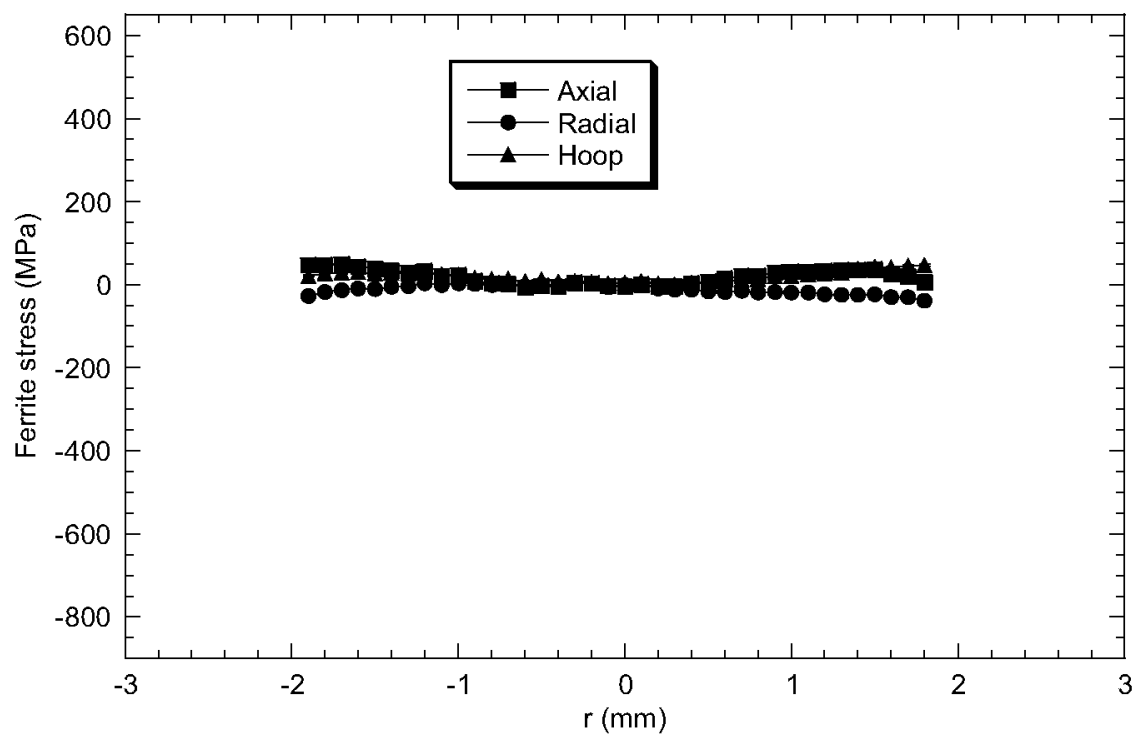

Fig. 8 Residual stress profiles obtained from the synchrotron measurements in the ferrite phase of the 'stabilized' steel rod (severely drawn material) [15]. Error bars are smaller than the symbols employed

samples of each type of steel, following the ISO recommendation [17], were tested. The average time to rupture was: $2.0 \mathrm{~h}$ for the as-drawn wires, $4.2 \mathrm{~h}$ for the stabilized wires, and $12.0 \mathrm{~h}$ for the rolled wires $[\mathbf{1 3}]$. It has been shown that the residual stresses in the surface region of the rod (up to a depth of approximately $0.75 \mathrm{~mm}$ ) are the key parameter controlling the time to rupture in this test [13]. Consequently, the durability in aggressive environments can be significantly improved with a careful 'design' of the residual stress profile.

\section{SUMMARY AND FINAL COMMENTS}

Synchrotron strain scanning is a very powerful technique for experimental strain analysis in cold-drawn steel rods. Residual strain/stress profiles with high spatial resolution can be obtained together with penetration depths comparable to neutrons (i.e. full diameter measurements in a $18 \mathrm{~mm}$ diameter steel rod). The use of high-energy white beam configuration is very useful in this case. 


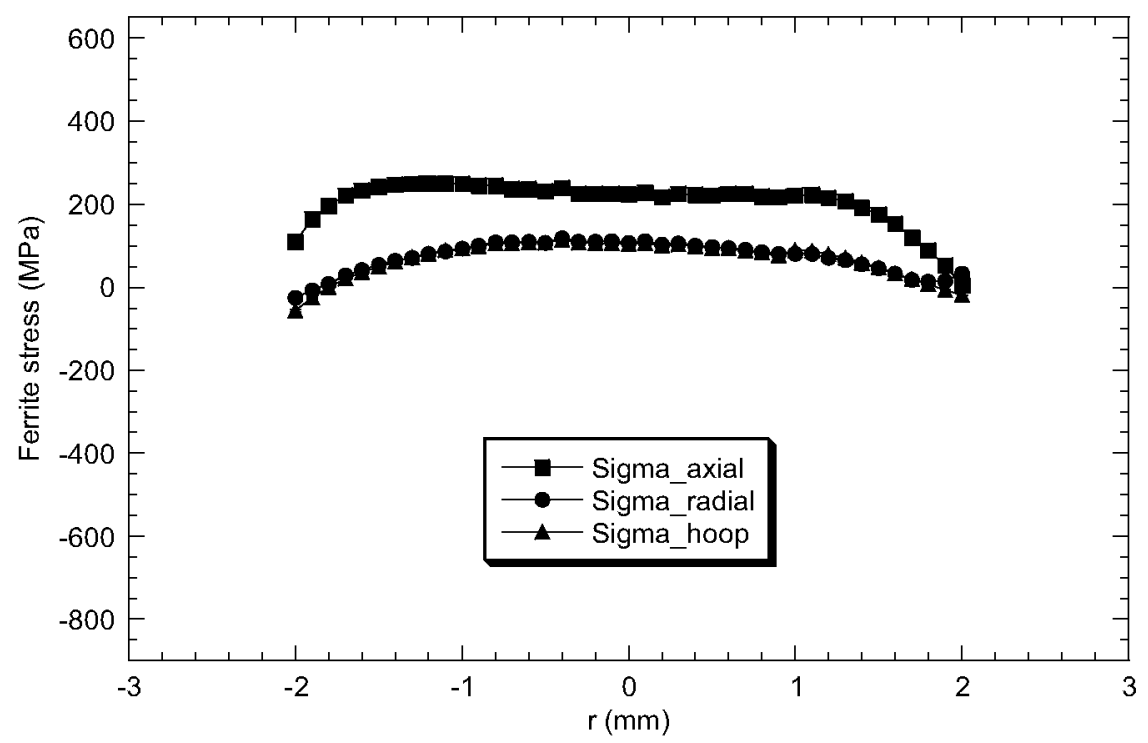

Fig. 9 Residual stress profiles obtained from the synchrotron measurements in the ferrite phase of the 'rolled' steel rod (severely drawn material) [15]. Error bars are smaller than the symbols employed

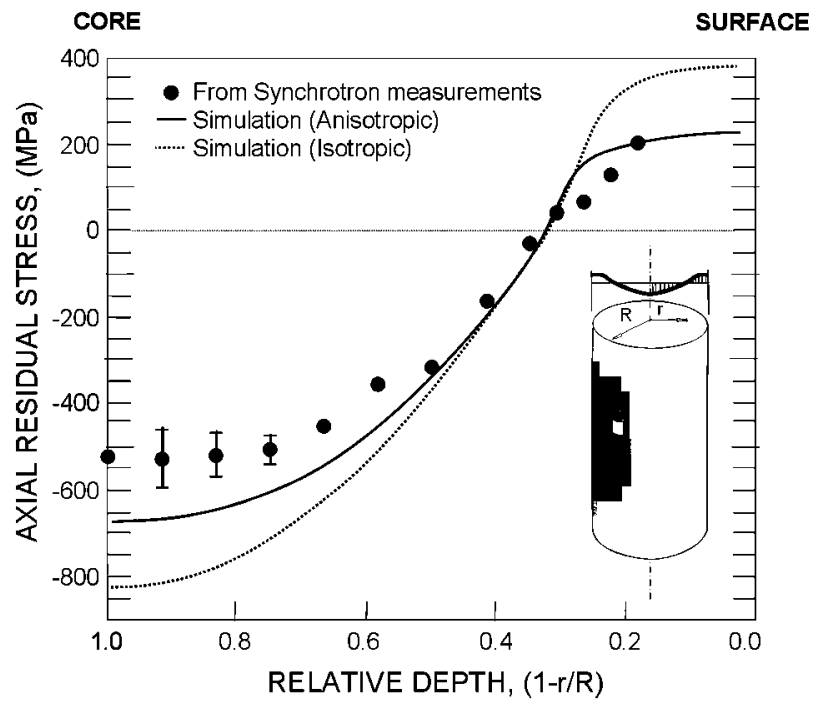

Fig. 10 Axial residual macrostresses in the lightly drawn steel rod. A comparison is shown between the values of the finite element simulation and those computed from the synchrotron measurements $[\mathbf{1 0}]$. The results of the finite element simulation when the anisotropy is not considered are also shown (Reprinted from [10] with permission from Elsevier.)

Residual stress profiles were obtained in two materials with a different degree of cold-drawing: lightly and severely drawn. Despite the difference in the values of the true strain achieved in both materials, the resulting stress profiles are quite similar. It seems that the cold-drawing process erases the previous residual stress history of the material and only the final drawing step determines the resulting residual stress profile.

Residual stresses computed by numerical simulation were compared with experimental results obtained with synchrotron strain scanning. The agreement is good when both phases of the pearlitic steel rod (i.e. ferrite and cementite) can be measured by diffraction. Despite the low volume fraction of cementite (around 10 per cent) its contribution to the macro-residual stress cannot be ignored if accurate values are needed.

A detailed knowledge of the residual stress profiles may help to improve the performance of colddrawn steel rods. Residual stresses are not always harmful. It was demonstrated that compressive residual stresses at the rod surface enhance the durability in aggressive environments. In fact, the time to rupture of the rods in the FIP ammonium thyocianate test can be multiplied by six with an adequate residual stress state.

Consequently, the use of synchrotron radiation has been very useful to improve existing cold-drawing processes and post-drawing treatments by using high-resolution strain scanning.

\section{ACKNOWLEDGEMENTS}

The authors gratefully acknowledge the support of the Ministerio de Educación, by means of the projects CICYT MAT 2003-00836, ENE2005-06478/CON and BIA2008-06705-C02-01. The present work was conducted within the framework provided by the projects DUMEINPA, sponsored by the Comunidad 


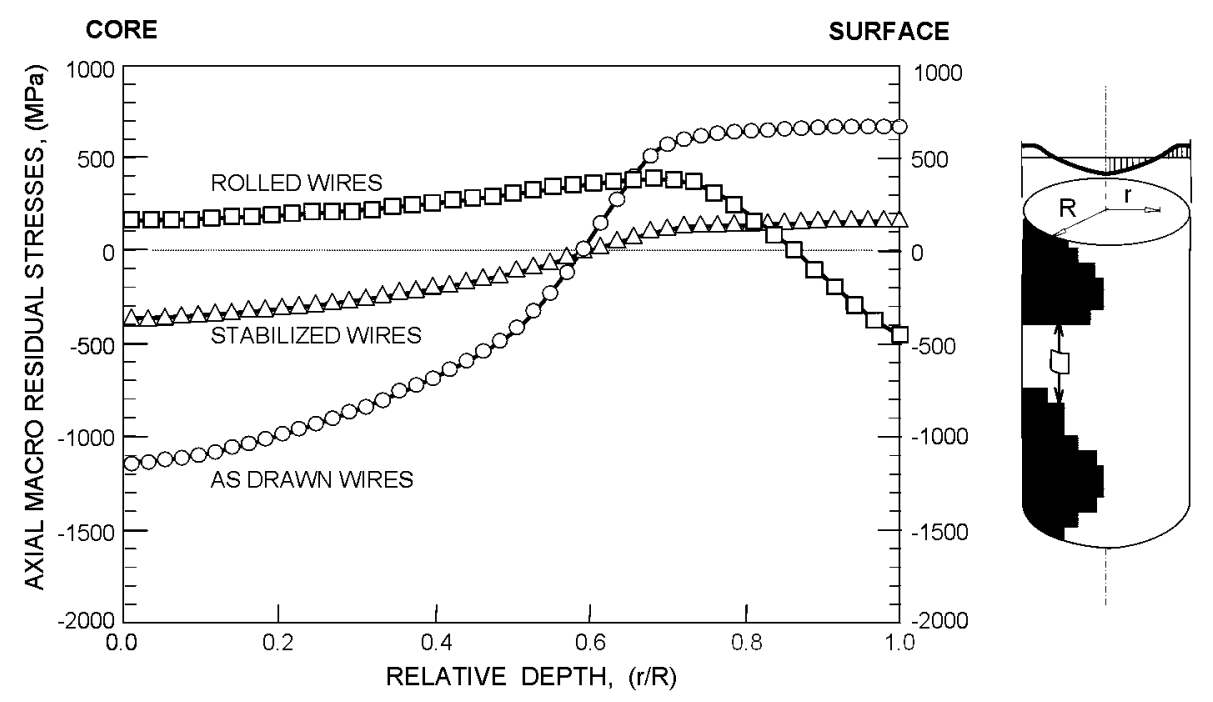

Fig. 11 Typical macro residual stress profiles (in the axial direction) along the wire diameter for as-drawn, stabilized, and rolled wires, as calculated by finite element simulations (Reproduced with kind permission from Springer Science+Business Media [6].)

de Madrid, Spain, and SEDUREC, integrated in the Spanish national research programme CONSOLIDERINGENIO 2010. The authors are indebted to María Martínez, Mar García, Federico Mompeán, Axel Steuwer, Jens Altenkirch, Mathew Peel, Thomas Buslaps, and Vladimir Luzin for their contribution to the neutron and synchrotron measurements, and Dr Luis Caballero for the stress corrosion tests. Help and useful comments from Mr Luis del Pozo, Emesa Trefilerías, and Mr Javier del Río, from Bekaert, are also acknowledged.

(c) Authors 2011

\section{REFERENCES}

1 Embury, J. D. and Fisher, R. M. The structure and properties of drawn pearlite. Acta Metall., 1996, 14, $147-159$.

2 Langford, G. Deformation of pearlite. Metall. Trans., 1977, 8A, 861-875.

3 Taleff, E. M., Lewandowski, J. J., and Pourladian, B. Microstructure-property relationships in pearlitic eutectoid and hypereutectoid carbon steels. J. Ops $M g m t, 2002,54,25-30$.

4 Llorca, J. and Sánchez-Gálvez, V. Numerical determination of the influence of residual stresses on fatigue. Proceedings of the International Conference on Computational plasticity, Barcelona, 1987, pp. 1123-1136 (Pineridge Press Limited).

5 Elices, M., Maeder, G., and Sánchez-Gálvez, V. Effect of surface residual stress on hydrogen embrittlement of prestressing steels. Br. Corros. J., 1983, $18,80-81$.
6 Atienza, J. M., Ruiz-Hervias, J., Caballero, L., and Elices, M. Residual stresses and durability in cold drawn eutectoid steel wires. Met. Mater-Int., 2006, 13(2), 139-143.

7 Van Acker, K., Root, J., van Houtte, P., and Aernoudt, E. Neutron diffraction measurement of the residual stress in the cementite and ferrite phases of cold-drawn steel wires. Acta Mater., 1996, 44, 4039-4049.

8 Suzuki, T., Tomota, Y., Isaka, M., Moriai, A., Minakawa, N., and Morii, Y. Strength anisotropy and residual stress in drawn pearlite steel wire. ISIJ Int., 2004, 44, 1426-1430.

9 Martinez-Perez, M. L., Mompean, F. J., RuizHervias, J., Borlado, C. R., Atienza, J. M., GarcíaHernandez, M., Elices, M., Gil Sevillano, J., Peng, R. L., and Buslaps, T. Residual stress profiling in the ferrite and cementite phases of cold-drawn steel rods by synchrotron X-ray and neutron diffraction. Acta Mater., 2004, 52, 5303-5313.

10 Atienza, J. M., Ruiz-Hervías, J., Martínez-Perez, M. L., Mompeán, F. J., García-Hernandez, M., and Elices, M. Residual stresses in cold drawn pearlitic rods. Scripta Mater, 2005, 52, 1223-1228.

11 Ruiz-Hervias, J., Luzin, V., Prask, H., GnaeupelHerold, T., and Elices, M. Effect of thermo-mechanical treatments on residual stresses measured by neutron diffraction in cold-drawn steel rods. Mater. Sci. Engng, 2006, 435-436, 725-735.

12 Ruiz-Hervías, J., Atienza, J. M., Elices, M., and Oliver, E. C. Optimisation of post-drawing treatments by means of neutron diffraction. Mater. Sci. Engng A, 2008, 480(1-2), 439-448.

13 Elices, M., Caballero, L., Valiente, A., RuizHervias, J., and Martín, A. Hydrogen embrittlement of steels for prestressing concrete: the FIP and DIBt tests. NACE Corros. J., 2008, 64, 164-174. 
14 Ruiz, J., Atienza, J. M., and Elices, M. Residual stresses in wires: influence of wire length. J. Mater. Engng Perform., 2003, 12, 480-489.

15 Ruiz-Hervias, J., Steuwer, A., Altenkirch, J., Peel, M., Buslaps, T., and Elices, M. Residual strain scanning in cold-drawn eutectoid rods by high-energy synchrotron radiation. In Proceedings of the Third Meeting of the Spanish Synchrotron Users Association, 9-11 July 2007, Jaca, Spain.
16 Atienza, J. M. Residual stresses in cold drawn steel wires. PhD Thesis, Universidad Politecnica de Madrid, Spain, 2001 (in Spanish).

17 ISO (International Organization for Standardardization) ISO/DIS 15630.3: Steel for the reinforcement and prestressing of concrete. test methods. Part 3: prestressing steel, 2007, Section 10. ISO, Geneva. 